\title{
Massive clusters as seen by Spitzer
}

\author{
B. R. Brandl ${ }^{1}$, L. K. Townsley ${ }^{2}$, E. Churchwell ${ }^{3}$, S. Carey ${ }^{4}$, \\ H. Zinnecker ${ }^{5}$, P. Massey ${ }^{6}$, J. R. Stauffer ${ }^{4}$, R. Hurt ${ }^{4}$ and J. R. Houck ${ }^{7}$ \\ ${ }^{1}$ Leiden University, P.O. Box 9513, 2300 RA Leiden, The Netherlands \\ ${ }^{2}$ Dept. of Astronomy, Penn State, 525 Davey Lab., University Park, PA 16802, USA \\ ${ }^{3}$ Dept. of Astronomy, 475 N. Charter St., Madison, WI 53706-1582, USA \\ ${ }^{4}$ Caltech Spitzer Science Center, 314-6, Pasadena, CA 91125, USA \\ ${ }^{5}$ Astrophysikalisches Institut Potsdam, An der Sternwarte 16, 14482 Potsdam, Germany \\ ${ }^{6}$ Lowell Observatory, 1400 W. Mars Hill, Flagstaff, AZ 86001, USA \\ ${ }^{7}$ Cornell University, Astronomy Department, Ithaca, NY 14853, USA
}

\begin{abstract}
The Spitzer Space Telescope has delivered impressive infrared images of numerous low-mass star forming regions at unprecedented sensitivity. In this paper we focus on their high-mass counterparts, the most massive HiI regions in the Local Group: NGC 3603 (MW), W49A (MW), 30 Doradus (LMC), NGC 346 (SMC), and NGC604 (M33). The deep mid-IR images, taken with IRAC show the complex structure of the ISM, the interplay between ISM and stellar clusters, and reveal new sites of star formation. We compare the IRAC data with observations from Chandra and find good anti-correlations between the mid-IR and soft X-ray emission regions. We also investigate the commonalities and differences between the giant HII regions NGC 604 and 30 Doradus.
\end{abstract}

Keywords. stars: formation, ISM: HII regions, ISM: dust, extinction, galaxies: star clusters, infrared: ISM

\section{Introduction}

We conducted deep Spitzer-IRAC imaging of the most massive HII regions in the Local Group to address the the fundamental question: What are the common properties of massive star-forming regions, and how do these properties vary with age and environment. The data presented here are part of a guaranteed time observing program of the IRS instrument team, which also includes follow-up spectroscopy but not as part of this paper. Table 1 gives an overview of the observations.

Note: this paper summarizes the presentation given at the IAU symposium \#227, and was largely based on the qualitative discussion of color composities between IRAC filter bands and IRAC-Chandra-VLA overlays. Unfortunately, neither the color overlays nor the discussion of the features revealed by these images can be reproduced here. However, more details and high-quality multi-color figures are provided in Brandl et al. (2005).

Table 1. Spitzer-IRAC Observing Parameters

\begin{tabular}{lcccc}
\hline Name & Obs. Date & $t_{\text {int }}$ & Map pos. & Dither pos. \\
NGC 3603 & Jan 2004 & $2 \times 12 \mathrm{~s}$ & & 12 \\
W49 & 20 Apr 2004 & $1 \times 12 \mathrm{~s}$ & & 24 \\
30 Doradus & 06 Dec 2003 & $1 \times 12 \mathrm{~s}$ & $4 \times 6$ & 3 \\
NGC 346 & 20 Apr 2004 & $2 \times 12 \mathrm{~s}$ & & 18 \\
NGC 604 & 28 Dec 2003 & $2 \times 12 \mathrm{~s}$ & & 18 \\
\hline
\end{tabular}

${ }^{a} 30$ Doradus has also been observed previously as part of the "early release observations" program on 6 Nov 2003. 


\section{The individual regions}

We mainly present images taken through the IRAC $6.5-9.4 \mu \mathrm{m}$ filter band, where the emission is dominated by the polycyclic aromatic hydrocarbon $(\mathrm{PAH})$ features at $7.7 \mu \mathrm{m}$ and $8.6 \mu \mathrm{m}$. Molecular gas in the boundary layers surrounding the HII regions is exposed to FUV radiation $(6-13.6 \mathrm{eV})$ and PAHs are photoelectrically heated. Thus, PAHs serve as an important tracer of star formation activity. However, one should keep in mind that the PAH emission detected in these images originates from the surfaces of these interfaces and is not a good tracer of column density.

\section{1. $W 49 A$}

$\mathrm{W} 49 \mathrm{~A}$ is one of the most luminous and prolific massive star formation regions in the disk of the Milky Way. About 40 separate UC HiI regions (O-subgroups or clusters) with sizes as small as $0.01 \mathrm{pc}$ are located within a region of about $55 \mathrm{pc}$ (Welch et al. 1987). The near simultaneous ignition of so many star formation regions over such a large volume of space is one of the great puzzles of modern astronomy. This phenomenon is observed not only in W49A but also in the other giant star formation regions discussed here. Recently, Alves \& Homeier (2003) have identified 100 O-star candidates only about 2 parsecs away from the UCHIIRs, most of which are confined to four clusters identified at NIR bands. Figure 1 shows the Spitzer-IRAC $8 \mu \mathrm{m}$ gray-scale image of W49A.

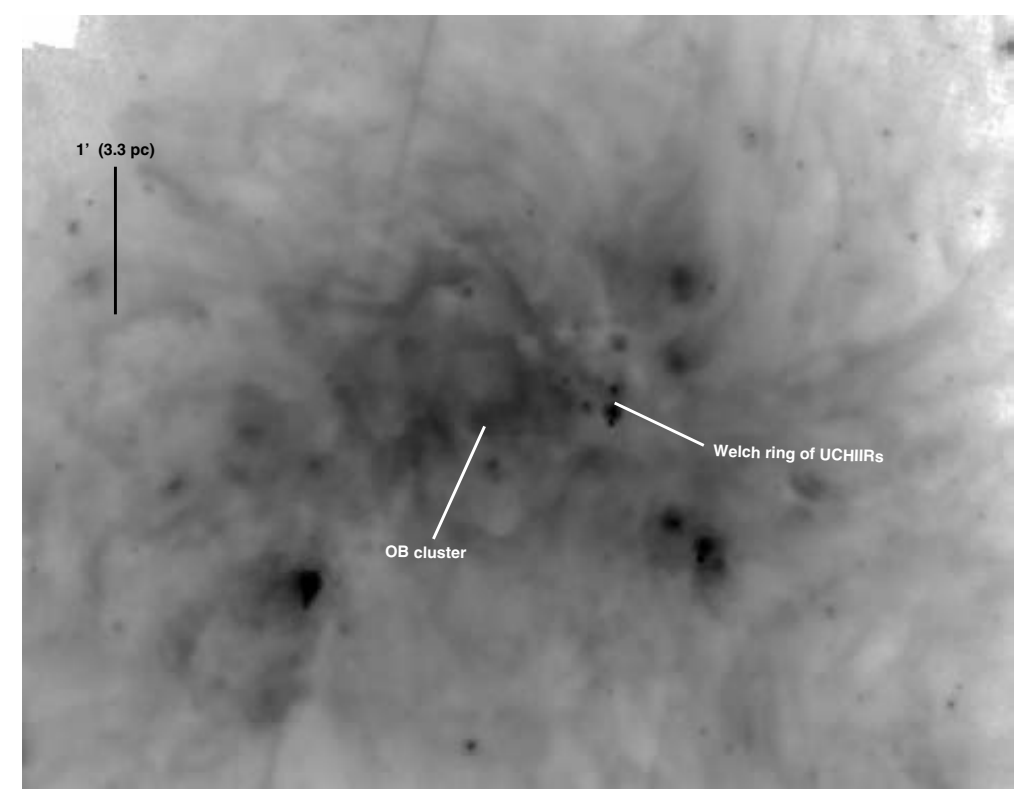

Figure 1. Spitzer-IRAC $8 \mu \mathrm{m}$ gray-scale image of W49A. An improved color version of this figure will be provided in Brandl et al. (2005).

With a possible connection between the masive OB cluster and the nearby ring of UCHIIRs, likely resulting from triggered star formation, W49A may serve as a template for more luminous starbursts. If UCHIIRs do exist in starbursts and can survive for a significant $\left(10^{6} \mathrm{yrs}\right)$ period they may help to solve the discrepancy between the observed fine-structure line ratios (e.g., $[\mathrm{NeIII}] /[\mathrm{NeII}]$ ) and the total luminosity of a starburst region (Thornley et al. 2000). 


\section{2. $N G C 3603$}

NGC 3603 is the only massive, Galactic HiI region whose ionizing central cluster can be studied at optical wavelengths due to only moderate (foreground) extinction. In comparison, NGC 3603 with its more than $50 \mathrm{O}$ and WR stars, has about 100 times the ionizing power of the Orion Trapezium cluster. With a bolometric luminosity $L_{\mathrm{bol}} \geqslant 10^{7} L_{\odot}$, NGC 3603 has about $10 \%$ of the luminosity of 30 Doradus and looks in many respects very similar to its stellar core R136 (Brandl et al. 1996). Figure 2 shows the Spitzer-IRAC $8 \mu \mathrm{m}$ gray-scale image of NGC 3603.

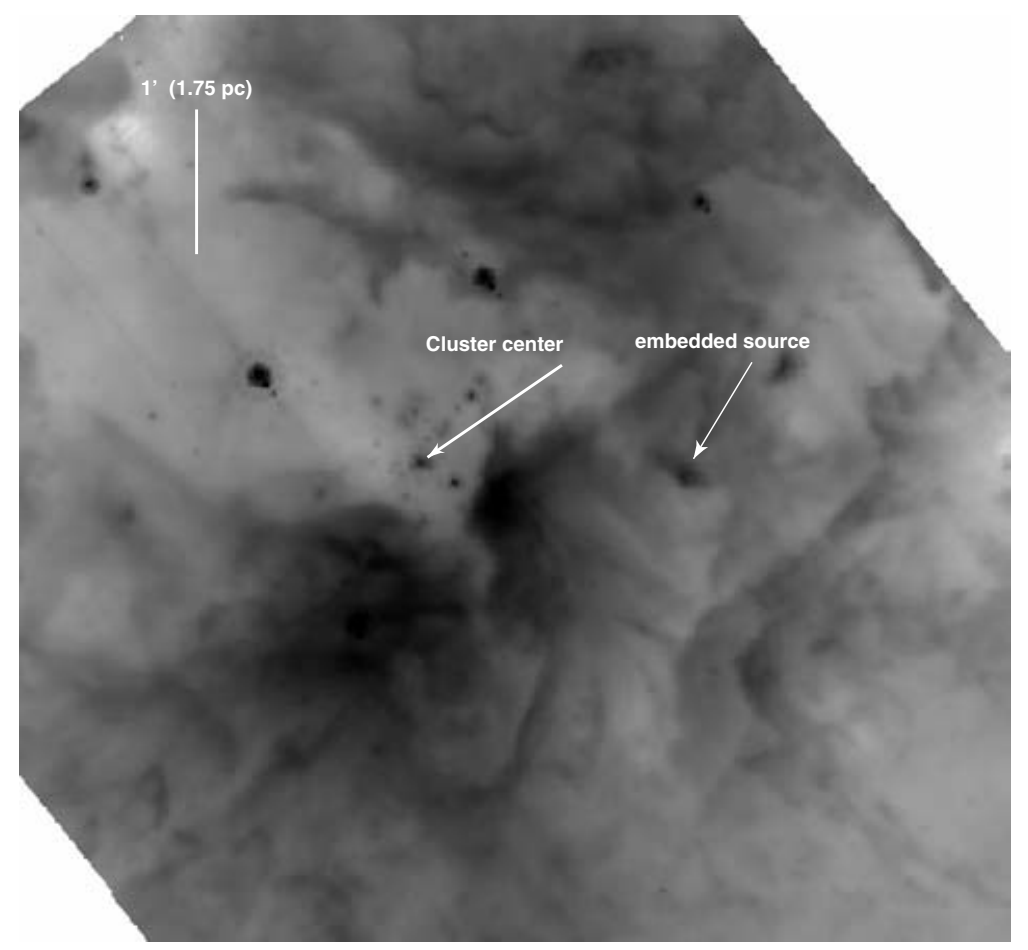

Figure 2. Spitzer-IRAC $8 \mu \mathrm{m}$ gray-scale image of NGC 3603. An improved color version of this figure will be provided in Brandl et al. (2005).

The molecular cloud surrounding NGC 3603 is highly asymmetric and extends almost 20 parsec toward the southwest of the cluster core. Several pillars of 2 parsec lengths and bow shock-like structures are detected. The IRAC image also reveals multiple, previously undetected and heavily embedded sources, preferentially near the tips of the pillars and along the filaments.

\subsection{Doradus}

At a distance of only about $53 \mathrm{kpc} 30$ Doradus is the closest massive star forming region and the best studied template of a starburst described in over 3000 papers to date. Table 2 lists its constituents and sizes. According to Walborn \& Blades (1997) NGC 2070 consists of several distinct, young stellar generations. With about 40 stars of spectral type O3 R136 is the densest concentration of very massive stars known (Massey \& Hunter 1998).

Figure 3 shows the complex filamentary structure of the 30 Dor region, extending over almost 500 parsecs. The central stellar cluster, R136, is located to the southeastern rim of the most massive gas cloud. 
Table 2. Designations related to the 30 Dor complex

\begin{tabular}{llcr}
\hline Name & Class & angular $\varnothing$ & linear $\varnothing$ \\
LMC & galaxy & $5^{\mathrm{O}}$ & $5000 \mathrm{pc}$ \\
30 Dor region & complex & $1^{\mathrm{O}}$ & $1000 \mathrm{pc}$ \\
30 Dor nebula & Hil region & $15^{\prime}$ & $200 \mathrm{pc}$ \\
NGC 2070 & stellar cluster & $3^{\prime}$ & $40 \mathrm{pc}$ \\
R136 & stellar core & $10^{\prime \prime}$ & $2.5 \mathrm{pc}$ \\
\hline
\end{tabular}

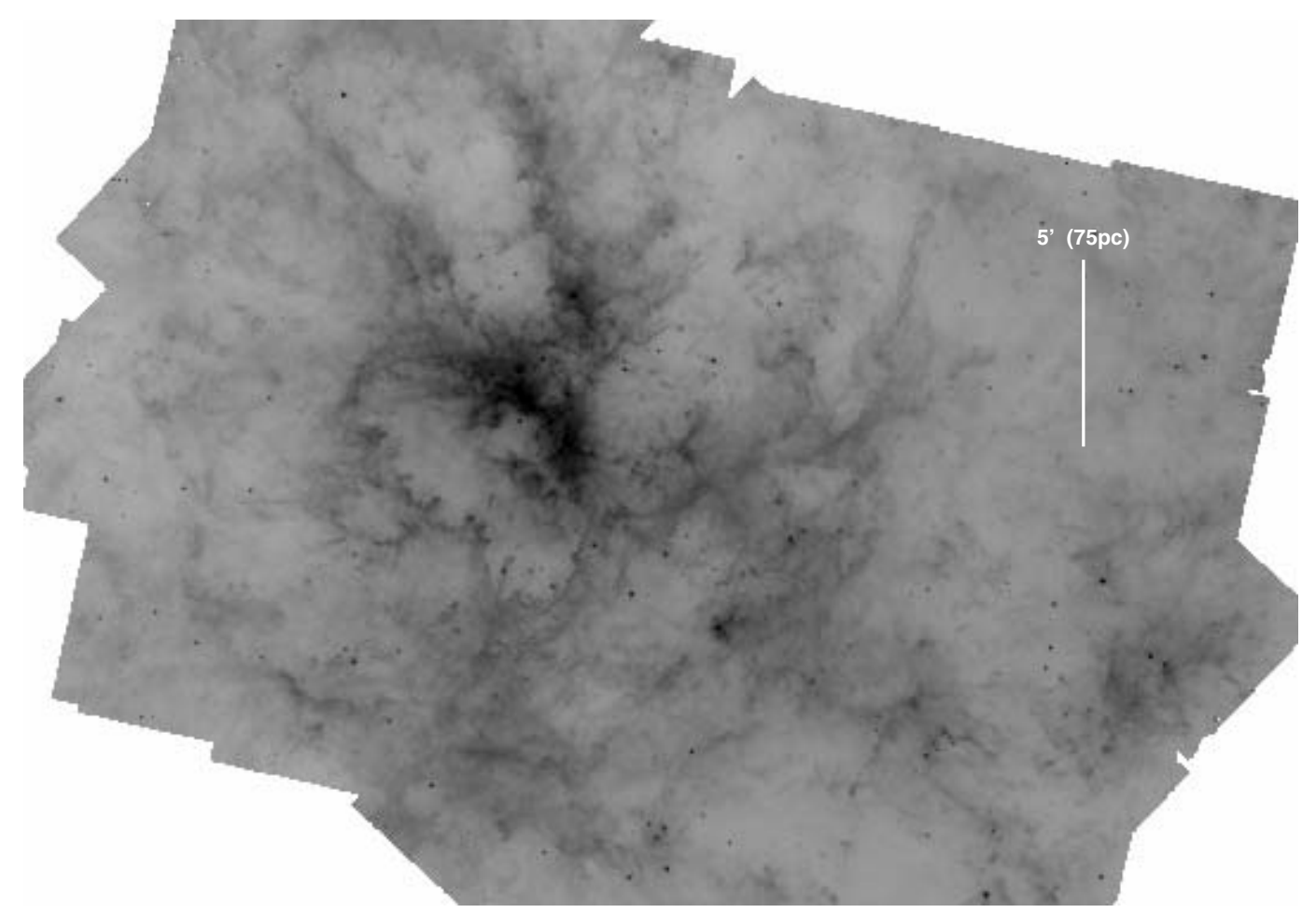

Figure 3. Spitzer-IRAC $8 \mu \mathrm{m}$ gray-scale image of 30 Dor. An improved color version of this figure will be provided in Brandl et al. (2005).

X-ray studies detect the presence of past supernovae through the shocks in their extended remnants. Powerful stellar winds in $\mathrm{OB}$ associations blow superbubbles 50 $100 \mathrm{pc}$ in size and fill them with hot, X-ray-emitting plasma with luminosities of $L_{x} \sim$ $10^{35-36} \mathrm{ergs} / \mathrm{s}$ and temperatures around 1-10 MK (Chu \& Mac Low 1990). This emission from each superbubble is quite faint $\left(L_{x}=10^{32-34} \mathrm{ergs} / \mathrm{s}\right)$, and essentially unobservable in GEHRs with today's technology, but combined together they reach $L_{x}=10^{37} \mathrm{ergs} / \mathrm{s}$ for the entire 30 Dor Nebula. Figure 4 shows the anti-correlation between X-ray and mid-IR emission.

\section{4. $N G C 346$}

NGC 346 (or N66) is the largest HiI region in the SMC, ionized by a "plethora of O stars" (Massey et al. 1989) with a present day mass function extending up to about 80 $M_{\odot}$ (Massey et al. 2005). It has 60 times the $\mathrm{H}_{\alpha}$ luminosity of the Orion nebula, and is also the region with the lowest metallicity $\left(\sim 1 / 10 Z_{\odot}\right)$ within our sample. 


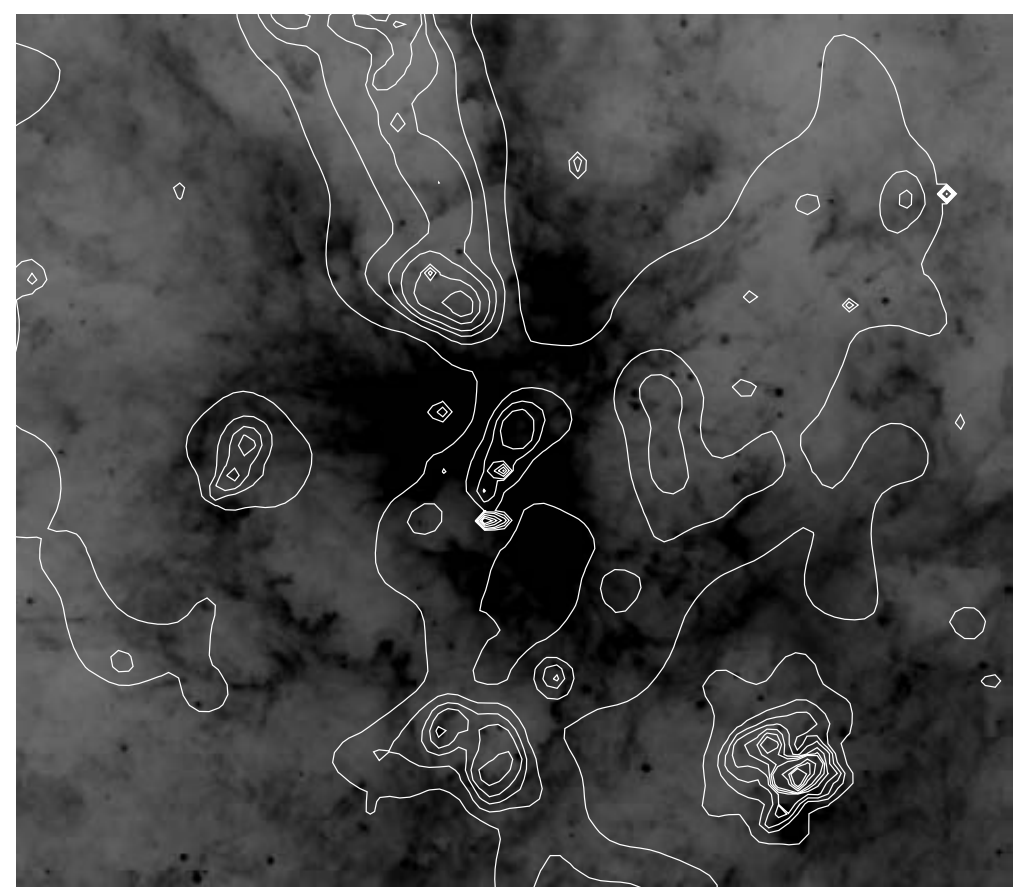

Figure 4. Spitzer-IRAC $8 \mu \mathrm{m}$ gray-scale image of 30 Dor with the soft X-ray emission from Chandra overlaid as contour lines. An improved color version of this figure will be provided in Brandl et al. (2005).

Fig. 5 shows the filamentary structure of NGC 346 in the $8 \mu \mathrm{m}$ PAH emission, which correlates well with the optical B-band emission seen with HST-ACS (Nota et al. 2005). However, the warm dust, as traced by the MIPS $24 \mu \mathrm{m}$ emission (not shown here) is much more spherically centered on the core of the cluster. In comparison to the other massive HII regions the lack of a large reservoir of gas and dust is obvious. Nevertheless, the MIPS and IRAC images reveal several sites of still embedded star formation. In contrast to other massive HiI regions no X-ray superbubbles have been detected in NGC 346.

\section{5. $N G C 604$}

At a distance of about $840 \mathrm{kpc}$, NGC 604 is the second most luminous HiI region in the Local Group. Its $\mathrm{H}_{\alpha}$ luminosity is only about $1 / 4$ of that of 30 Dor but it is still 450 times more luminous than the Orion nebula (Hunter 1999).

Although the H II regions discussed here all contain hundreds of massive stars, only 30 Doradus is dominated by a dense "supercluster", similar to what a small globular cluster was like when it was young (Hunter 1999). The surface density of stars with $M_{V}<-4$ (in terms of stars $\mathrm{pc}^{-2}$ ) in the NGC 604 complex is similar to that of other OB associations in the Milky Way and Magellanic Clouds, while the density of stars with that of the R136 cluster is two orders of magnitude higher. Nevertheless, the IRAC images of both 30 Dor and NGC 604 show very similar sizes and large scale structures of the ISM, and about the same infrared luminosity.

\section{Some conclusions}

We have presented deep images of a sample of the most massive HiI regions within the Local Group. The Spitzer-IRAC data reveal the complex structure of these regions 


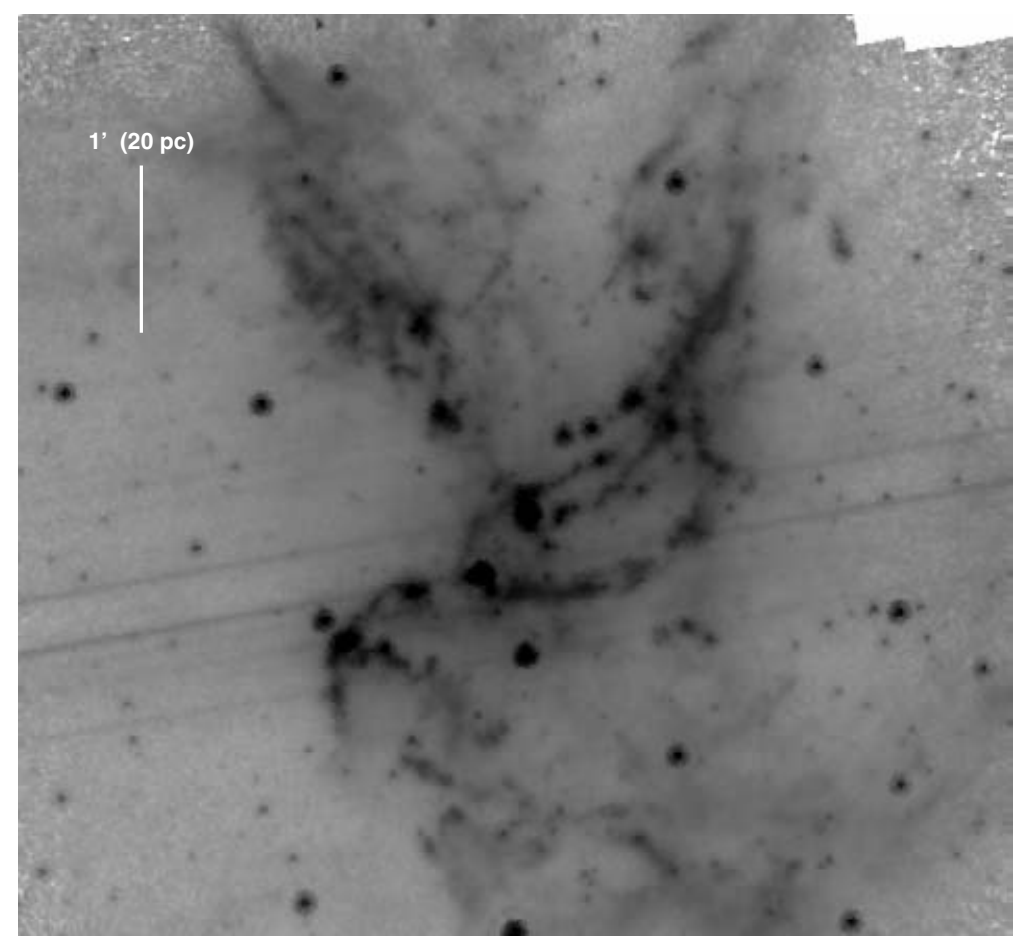

Figure 5. Spitzer-IRAC $8 \mu \mathrm{m}$ gray-scale image of NGC 346. An improved color version of this figure will be provided in Brandl et al. (2005).

in terms of the complexity and extent of the ISM, and the interplay between stars and gas.

Most massive clusters seem to be preferentially located near the edges of GMCs, not in their centers. The HII regions contain multiple stellar generations with supernova remnants (indicated by the X-ray superbubbles), main sequence early-type O-stars, and still embedded sources, all coinciding within the same region.

The large scale morphology of the ISM in massive HiI regions is dominated by filaments and cavities, and appears to be rather independent of their stellar cluster distributions or densities. Alternatively, the observed morphology of 30 Dor has been defined by its previous generation of clusters and is not (yet) affected by its most luminous central cluster, R136.

Last but not least, the observations demonstrate the power of Spitzer mid-IR imaging over several magnitudes in brightness and distance. Next we will use follow-up spectroscopy with the Spitzer-IRS to study the physical properties of these regions in more detail.

\section{Acknowledgements}

This work is based [in part] on observations made with the Spitzer Space Telescope, which is operated by the Jet Propulsion Laboratory, California Institute of Technology under NASA contract 1407. Support for this work was provided by NASA through Contract Number 1257184 issued by JPL/Caltech. 


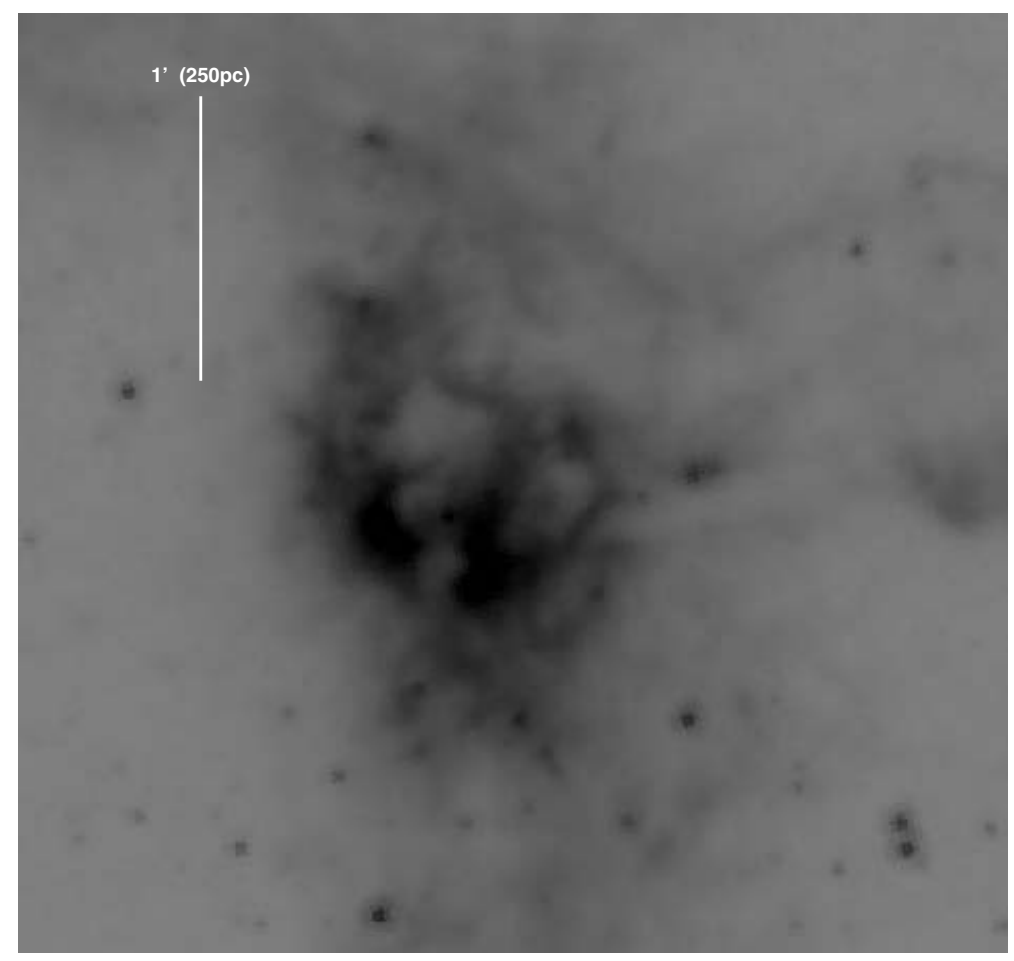

Figure 6. Spitzer-IRAC $8 \mu \mathrm{m}$ gray-scale image of NGC 604. An improved color version of this figure will be provided in Brandl et al. (2005).

\section{References}

Alves, J., Homeier, N. 2003, ApJL, 589, L45

Brandl, B. R. et al. 1996, ApJ, 466, 254

Brandl, B. R. et al. 2005, ApJ, submitted

Chu, Y.-H. \& Mac Low 1990, ApJ, 365, 510

Hunter, D. A. 1999, in Wolf-Rayet Phenomena in Massive Stars and Starburst Galaxies, IAU 193, ed. K. A. van der Hucht, G. Koenigsberger, \& P. R. J. Eenens (San Francisco: ASP), 418

Massey, P., Parker, J. W., \& Garmany, C. D. 1989, AJ, 98, 1305

Massey \& Hunter 1998, ApJ, 493, 180

Massey, P., Puls, J., Pauldrach, A. W. A., Bresolin, F., Kudritzki, R. P., \& Simon, T. 2005, ApJ, submitted

Nota, A. et al. 2005, http://hubblesite.org/newscenter/newsdesk/archive/releases/2005/04/

Thornley, M. D., Schreiber, N. M. F., Lutz, D., Genzel, R., Spoon, H. W. W., Kunze, D. \& Sternberg, A., 2000, ApJ, 539, 641

Walborn, N.R. \& Blades, J.C. 1997, ApJS, 112, 457

Welch, W. J., Dreher, J. W., Jackson, J. M., Tereby, S., \& Vogel, S. N. 1987, Science, 238, 1550 